\title{
Microbiology Susceptibility Collected Duration
}

National Cancer Institute

\section{Source}

National Cancer Institute. Microbiology Susceptibility Collected Duration. NCI Thesaurus.

Code C162151.

Collected duration of a microbiology susceptibility event or finding. 